\title{
MODELAGEM TERMODINÂMICA E CINÉTICA DE PRECIPITAÇÃO DE SAIS NA PRESENÇA DE GLICOL
}

\author{
C. M. S. FIGUEIREDO ${ }^{1,}$, A. G. BARRETO Jr ${ }^{1}$, F. W. TAVARES ${ }^{1}$ \\ Universidade Federal do Rio de Janeiro, Departamento de Engenharia Química \\ E-mail para contato: camilsenna@gmail.com
}

\begin{abstract}
RESUMO - A deposição de incrustações em reservatórios e plataformas de petróleo é um problema comum na produção de óleo e gás, trazendo sérios problemas econômicos e operacionais. O monoetilenoglicol (MEG), utilizado como inibidor de formação de hidratos, pode levar a diminuição da solubilidade dos sais, influenciando no processo de precipitação e, consequentemente, incrustações. Diversos estudos termodinâmicos foram realizados, a fim de se determinar as condições favoráveis à precipitação de sais, porém, a determinação da cinética de precipitação é um fator mais complexo e que ainda requer intenso trabalho experimental e de modelagem matemática. Mais raros, e não menos importantes, são os estudos sobre a dinâmica de dissolução de sais precipitados. Método experimental para determinar taxa de dissolução de sais inorgânicos usando apenas uma câmera fotográfica e uma proposta de análise dos resultados são mostrados neste trabalho. Como exemplo, foram obtidos dados de dissolução de $\mathrm{NaCl}$ e proposto um modelo para descrever o comportamento dinâmico em diferentes condições de temperatura e concentração de MEG.
\end{abstract}

\section{INTRODUÇÃO}

A deposição de incrustações em reservatórios e plataformas de petróleo é um problema comum na produção de óleo e gás, trazendo sérios problemas econômicos e operacionais. Segundo Mackay (2003), a formação de incrustações em poços produtores ocorre principalmente devido à redução de temperatura e/ou aumento de concentração de sais. $\mathrm{O}$ aumento da concentração de sais pouco solúveis é causado, geralmente, por evaporação de solvente em processo de redução acentuada de pressão da tubulação ou vaso.

A água de formação (água do reservatório) contém, usualmente, quantidades significativas de cátions divalentes como $\mathrm{Ca}^{2+}, \mathrm{Mg}^{2+}, \mathrm{Ba}^{2+} \mathrm{e} \mathrm{Sr}^{2+}$. Estes podem interagir com ânions, como $\mathrm{SO}_{4}{ }^{2-}$ e $\mathrm{CO}_{3}{ }^{2-}$, presentes na água do mar (água de injeção), injetada para recuperação secundária de petróleo, gerando a precipitação de sais insolúveis (incrustações).

Os locais mais susceptíveis à formação de carbonatos $\left(\mathrm{CaCO}_{3}, \mathrm{FeCO}_{3}, \mathrm{SrCO}_{3}\right)$ são os equipamentos de superfície localizados nas plataformas, nos quais ocorrem as maiores quedas de pressão, na elevação do fluido até a superfície, através das colunas de produção (Mackay, 2003). Estas variações de condições operacionais, aliadas às variações de temperatura e ao alto tempo de residência nos vasos separadores, são as causas para a formação de precipitados. Os tipos de precipitado usualmente formado em processos de produção de petróleo são os carbonatos e sulfatos. 
Durante o processo de produção de petróleo e gás, diversos produtos químicos tais como inibidores de corrosão, inibidores de formação de hidratos, antiaglomerantes, e desemulsificantes são adicionados ao sistema, a fim de estimular e favorecer a produção. Contudo, alguns desses produtos, quando em contato com os íons presentes na água de formação podem levar a diminuição da solubilidade dos sais, influenciando no processo de precipitação. Uma prática da indústria de petróleo, para evitar a formação de hidratos durante a etapa de escoamento da mistura do poço de produção até a plataforma, é a injeção de inibidores de formação de hidratos na cabeça do poço. Os inibidores podem ser termodinâmicos ou cinéticos, em quantidades que variam de acordo com as condições de temperatura e pressão do poço (Chapoy et al., 2012). O Monoetilenoglicol (MEG) é o inibidor termodinâmico mais utilizado na indústria de petróleo. Porém, segundo Sandengen (2006), a presença de MEG em solução altera as atividades dos íons presentes na fase aquosa, diminuindo a solubilidade da maioria dos sais.

Diversos estudos de condições de equilíbrio termodinâmico foram realizados, a fim de se determinar as condições de precipitação de sais, tanto em condições operacionais submarinas quanto em condições de superfície. No entanto, é importante determinar a dinâmica de precipitação. A cinética de precipitação é um fator mais complexo e que requer intenso trabalho experimental e de modelagem matemática. Um ponto importante e pouco explorado é a cinética de dissolução de sais. Mecanismos de limpeza são pouco explorados e pouco estudados pela falta de informação e estudo do processo de dissolução de sais precipitados.

Neste trabalho, os fenômenos fisicoquímicos envolvidos na dissolução de sais inorgânicos em meio aquoso em condições que simulam processos de produção de petróleo são avaliados, contemplando tanto o tratamento termodinâmico, para descrever condições de equilíbrio, quanto a cinética de dissolução destes precipitados.

\section{METODOLOGIA}

\subsection{Etapa Experimental}

Os seguintes reagentes foram utilizados para realização do procedimento experimental: Água deionizada em sistema Milli-Q; Monoetilenoglicol: VETEC Química LTDA; Pureza 99,5\% e NaCl: VETEC Química LTDA; Pureza 99\%. As soluções foram preparadas individualmente, pesando-se em balança analítica a massa da solução de MEG e de água a fim de se atingir a concentração final desejada. A água Mili-Q era colocada previamente em banho de ultrassom à $60^{\circ} \mathrm{C}$, durante 3 horas. As massas de $\mathrm{MEG}$ e água pesadas eram em seguida transferidas para o vaso reacional do Masterview, tendo a preocupação de evitar a entrada de oxigênio no sistema. A solução era, então, homogeneizada utilizando-se agitador tipo hélice.

Os experimentos de dissolução foram realizados utilizando o aparato experimental demonstrado na Figura 1. 


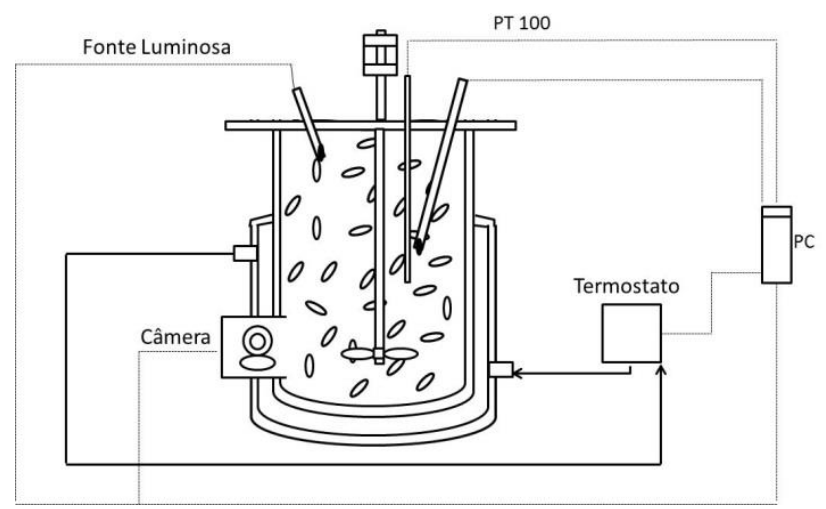

Figura 1 - Aparato experimental utilizado nos experimentos de dissolução, contendo câmera, fonte luminosa, termostato, agitador e sistema de aquisição de dados

Trata-se de um vaso reacional fabricado em vidro, com volume total de $100 \mathrm{ml}$, cujo controle de temperatura e agitação foi realizado através de banho termostático com controle de temperatura. Foi utilizada uma câmera, acoplada ao vaso e sem entrada de luz externa. Um LED foi introduzido no sistema como única fonte de luminosa.

O método de análise de imagem utilizado foi desenvolvido pelo grupo de pesquisa do Núcleo de Desenvolvimento de Processo e Análises Químicas em Tempo Real - Pólo de Xistoquímica/UFRJ. No método, utiliza-se uma webcam acoplada ao vaso reacional para o monitoramento de processos de dissolução. Imagens são aquisitadas em tempo real, através de software que monitora as variações nas componentes R (Red), G (Green) e B (Blue) pixel a pixel das imagens. O software permite a análise de uma área especifica da imagem capturada pela câmera, sendo suas coordenadas armazenadas, sendo calculado o valor médio dos valores de RBG. Alterações nos valores dessas componentes são detectadas pelo programa (Silva et al., 2013).
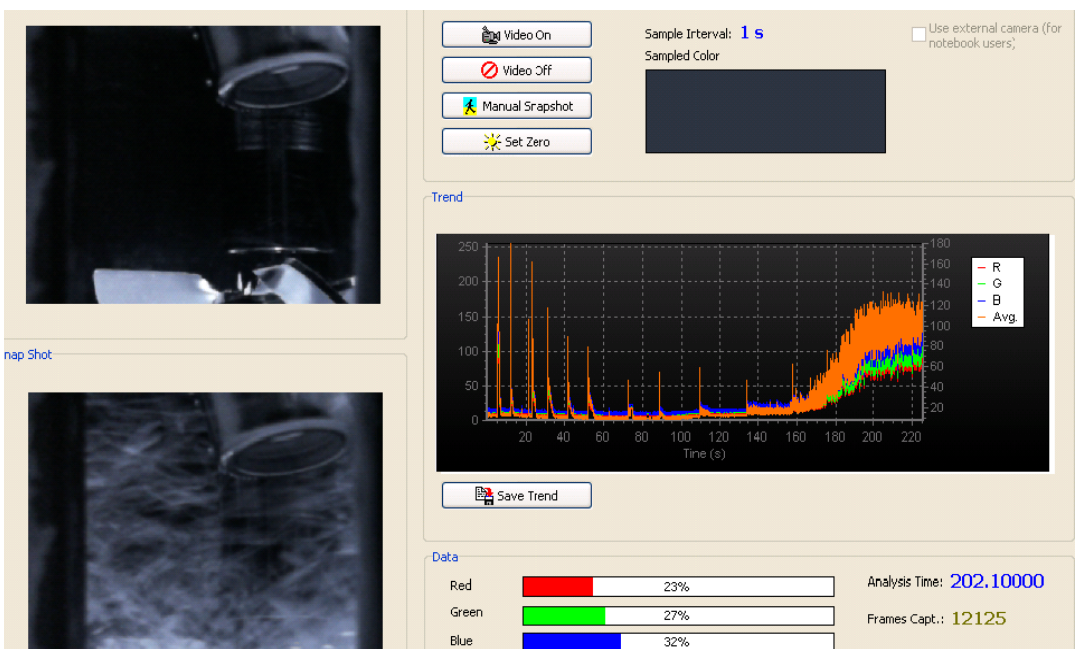

Figura 2 - Interface do software Masterview, mostrando as partículas em soluções, no lado esquerdo, e os picos de RGB como função do tempo, no lado direto.

Na Figura 2 observam-se duas fotos, do lado esquerdo, e um gráfico, do lado direito. A foto superior foi obtida antes do início do experimento e a imagem demonstra uma parte do vaso reacional contendo a solução líquida e o agitador. A foto inferior foi obtida após o início do experimento, e após a adição de sólidos à solução. O gráfico do lado direito representa a 
variação no valor de RGB após a adição de sólidos à solução ao longo do tempo. Após cada adição de $\mathrm{NaCl}$, o valor de RGB aumenta imediatamente, indicando a presença de sólidos em suspensão. Assim que a dissolução ocorre, o valor de RGB diminui assintoticamente.

Os experimentos de dissolução foram realizados nas temperaturas de 20,45 e $90^{\circ} \mathrm{C}$, a pressão ambiente e $300 \mathrm{rpm}$.

As massas de $\mathrm{NaCl}$ pesadas eram adicionadas ao vaso reacional, removendo-se a rolha de uma das aberturas existentes na tampa, utilizando-se de funil de vidro. Cada adição foi realizada após o desenvolvimento do perfil de dissolução, a partir, tanto da observação visual, quanto do registro dos valores de RGB ao longo do tempo.

\subsection{Modelagem Matemática}

Considerando as partículas como sendo cúbicas e a força motriz como sendo a diferença entre a constante de equilíbrio $\left(\mathrm{K}_{\mathrm{eq}}\right)$ e o produto de solubilidade $\left(\mathrm{K}_{\mathrm{ps}}\right)$, a definição da taxa de dissolução de uma partícula de massa $x$ é dada pela Equação (1).

$\dot{X}(x, t)=-6\left(\frac{x}{\rho}\right)^{2 / 3} k_{f}\left(K_{e q}-K_{p s}\right)$

sendo $\mathrm{k}_{\mathrm{f}}$ o coeficiente efetivo cinético de dissociação que inclui o coeficiente de transferência de massa, como descrito pela Equação (2).

$k_{f}=\frac{L R T}{\delta} \frac{1}{K_{e q}}=\frac{k T^{2}}{\eta^{\alpha} K_{e q}}$

Sendo k e $\alpha$ são parâmetros a serem estimados, T a temperatura da solução, $\mathrm{R}$ a constante de Boltzman, $\delta$ a espessura da camada difusional, $\eta$ a viscosidade da solução, $\rho$ a densidade da solução, $x$ a massa da partícula e L. o coeficiente de Onsage.

Considerando o aparato experimental descrito anteriormente, foi desenvolvido um balanço de massa global do sistema, um balanço de massa para a fase líquida e um balanço de massa para a fase sólida. O número de partículas na suspensão é descrito como um valor médio característico, isto é, todas as partículas apresentam a mesma massa em um dado instante $(\bar{x}(t))$. O balanço de massa para a fase sólida, uma vez que não há entrada, nem saída e nem geração, é dado pela igualdade entre o acúmulo e o que for consumido, conforme descrito pela Equação(3).

$\frac{d \bar{x}}{d t}=-k_{f}\left(K_{e q}-K_{p s}\right)\left(\bar{x}^{2 / 3}\right)+Q_{f}$

Em que $Q_{f}$ é dado pela Equação (4):

$Q_{f}=\frac{M_{f}}{\Delta t_{f}}$

Sendo $\bar{x}$ a massa de sólido (de tamanho representativo médio) em suspensão, $\mathrm{M}_{\mathrm{f}}$ a massa 
adicionada à solução, $\Delta \mathrm{t}_{\mathrm{f}} \mathrm{O}$ intervalo de tempo necessário para a adição de sal.

Analogamente, o balanço de massa para a fase líquida é dado pela Equação (5)

$$
\frac{d y}{d t}=k_{f}\left(K_{e q}-K_{p s}\right)(\bar{x})^{2 / 3}
$$

Em que y representa a concentração de soluto dissolvida no fluido.

A variação do volume do solvente devido ao processo de dissolução é dado pela Equação(6).

$\frac{d V}{d t}=-\frac{V}{\rho} \frac{\partial \rho}{\partial y} \frac{d y}{d t}$

Em que $V$ representa o volume total da solução líquida e $\rho$ representa a densidade da solução.

A solução dessas equações requer o uso de algumas equações auxiliares para definição da viscosidade (Equações (7 (10)) e da densidade (Equação (11)), ambas como função de temperatura, concentração de sal dissolvido e teor de MEG.

$$
\begin{aligned}
& \log \left(\eta_{M E G}\right)=-4,5448+\frac{417,05}{T-146,53} \\
& \log \left(\eta_{H 2 O}\right)=-4,5318+\frac{220,57}{T-149,39} \\
& \ln \left(\eta_{M E G+H 2 O}\right)=z_{M E G} \ln \left(\eta_{M E G}\right)+z_{H 2 O} \ln \left(\eta_{H 2 O}\right)+z_{M E G} z_{H 2 O}(-2,85993234+0,00062636 T) \\
& \log (\eta)=\ln \left(\eta_{M E G+H 2 O}\right)+z_{S a l}\left(\frac{1428,535}{T-95,9613}\right) \\
& \rho=0,99651-0,0297 T+0,12459 z_{z_{M E G}}+0,237546 z_{N a C l}-0,04193 z_{N a C l}^{2}-0,11162 z_{M E G} z_{N a C l}
\end{aligned}
$$

Sendo $z$ a fração mássica.

A massa de sólidos em suspensão $(\bar{x})$ e o volume da solução $(V)$ foram usados para gerar perfis temporais de RGB calculados a partir da Equação (12).

$R G B=\beta \frac{\bar{x}}{V}$

Sendo $\beta$ um parâmetro a ser estimado, representando o parâmetro de calibração. Como proposto neste trabalho beta é o coeficiente angular da reta que descreve a relação entre fração de sólidos (massa/volume) e RGB.

As equações do modelo matemático proposto neste trabalho foram integradas na forma 
de um sistema de equações diferenciais usando a subrotina Dassl implementada em Fortran.

Os parâmetros $\alpha, \beta$ foram estimados, a partir da minimização da função objetivo $F$ descrita pela Equação (13), utilizando o algoritmo enxame de partículas com $\mathrm{c}_{1}$ e $\mathrm{c}_{2}$ iguais a $1,5, \mathrm{w}_{\mathrm{i}}$ igual a 0,9 e $\mathrm{w}_{\mathrm{f}}$ igual a 0,5 . Foram usadas 150 partículas e 150 iterações.

$F(\theta)=\sum_{j}^{\text {Nexp }} \sum_{i=1}^{\text {Npontos }}\left(R G B^{\text {calc }}\left(\theta, t_{a d}\right)_{i, j}-R G B_{i, j}^{\text {exp }}\right)^{2}$

Onde $R G B_{i, j}^{\text {exp }}$ corresponde ao sinal do programa Masterview obtido em cada instante, definido pelo contador $\mathrm{i}$, em cada experimento $\mathrm{j} . t_{a d}{ }_{i, j}$ corresponde ao intervalo de tempo de adição de sal em cada instante, definido pelo contador $\mathrm{i}$, em cada experimento $\mathrm{j}$.

$R G B^{\text {calc }}(\boldsymbol{\theta})_{i, j}$ corresponde ao valor calculado de RGB, dado pela Equação (12), em cada instante definido pelo contador $\mathrm{i}$, em cada experimento j. $t_{a d}{ }^{\text {calc }}{ }_{i, j}$ corresponde ao intervalo de tempo de adição de sal em cada instante, definido pelo contador i, em cada experimento $\mathrm{j}$. $\boldsymbol{\theta}$ é o vetor de parâmetros formado por k, alfa, beta. Npontos é o número total de pontos adquiridos pelo Masterview e Nexp é o número de experimentos. Nadições é o número de adições de sal.

\section{RESULTADOS}

Os resultados obtidos através do método de análise de imagem demonstram que a adição de $\mathrm{NaCl}$ aumenta o sinal de RGB devido à mudança de cor causada pela presença de sólidos em suspensão. A solubilização do soluto diminui a concentração de sólidos em suspensão e esse processo é responsável pela redução nos valores de RGB. Um ponto a ser ressaltado é que o alargamento do pico de RGB deve-se à diminuição da taxa de dissolução de $\mathrm{NaCl}$, o que pode ser usado como uma métrica da condição de saturação.

Os dados experimentais gerados foram comparados com o modelo de dissolução desenvolvido neste trabalho, conforme Figura 3. As condições experimentais utilizadas em três experimentos distintos encontram-se na Tabela 1.

Tabela 1. Condições experimentais utilizadas nos experimentos de dissolução de $\mathrm{NaCl}$ em água/MEG.

$\begin{array}{cccc}\text { Experimento } & \text { Volume }(\mathrm{ml}) & \text { Temperatura }\left({ }^{\circ} \mathrm{C}\right) & \text { XMEG } \\ 1 & 80 & 20 & 0,125 \\ 2 & 86 & 20 & 0,581 \\ 3 & 86 & 45 & 0,581\end{array}$

A Figura 3 mostra dois experimentos de dissolução de $\mathrm{NaCl}$ em soluções contendo diferentes concentrações de MEG, a $20^{\circ} \mathrm{C}$. Comparando-se as primeiras adições em cada uma das soluções, observa-se que menor foi a taxa de dissolução do sal na solução contendo fração mássica de MEG igual a 0,581, quando comparada à solução contendo fração mássica de MEG igual a 0,125 . O mesmo comportamento foi observado durante as demais adições. $\mathrm{O}$ 
modelo proposto é capaz de representar esses dados experimentais, descrevendo satisfatoriamente as variações de comportamento.

Nota-se que o aumento da concentração de MEG influencia diretamente na viscosidade da solução e que tenderá a diminuir o coeficiente de transferência de massa (Equação (2)) e, consequentemente, reduzir a taxa de dissolução do $\mathrm{NaCl}$.

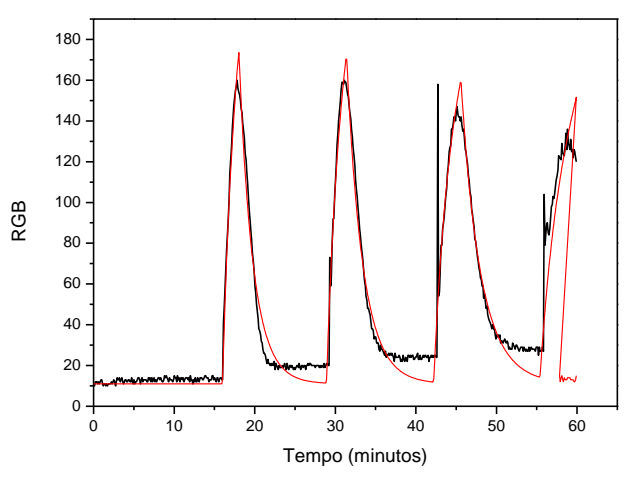

(a)

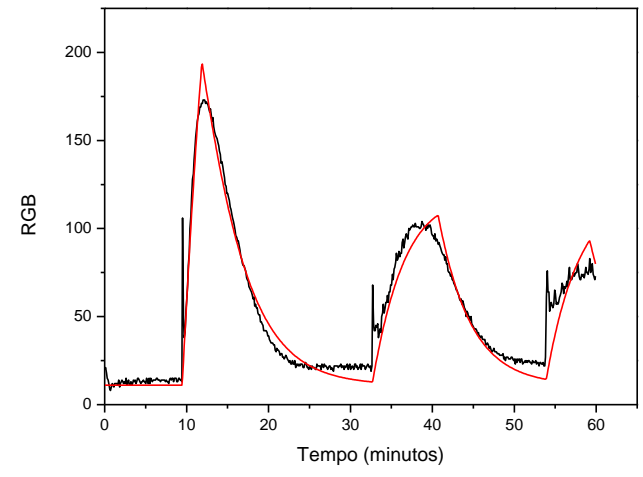

(b)

Figura 3. Comparação de valores de RGB calculados com o modelo proposto (linha vermelha) e os dados experimentais (linha preta) correspondentes ao experimento 1; (b) experimento 2 .

A Figura 4 mostra um experimento de dissolução de $\mathrm{NaCl}$ em solução de $\mathrm{MEG}$ e água, em duas temperaturas diferentes. Observa-se que, para uma mesma quantidade de $\mathrm{NaCl}$ adicionada inicialmente, menor a taxa de dissolução do sal a $20^{\circ} \mathrm{C}$ (maior a largura do pico, conforme mostrado na figura) quando comparado ao experimento realizado a $45^{\circ} \mathrm{C}$. $\mathrm{O}$ mesmo comportamento foi observado durante a segunda adição. $\mathrm{O}$ modelo proposto é capaz de representar satisfatoriamente o comportamento observado experimentalmente.

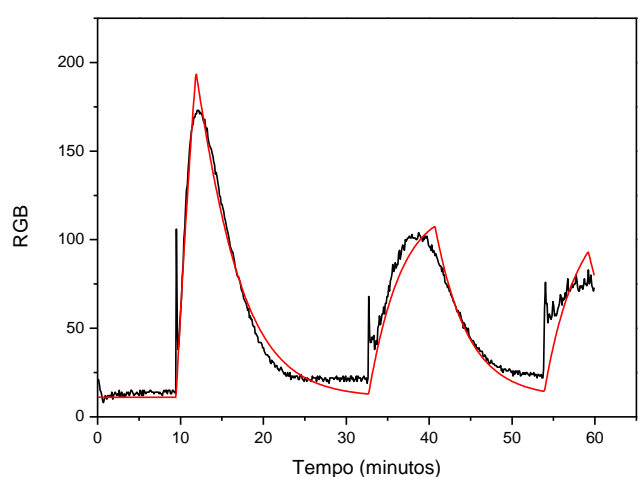

(a)

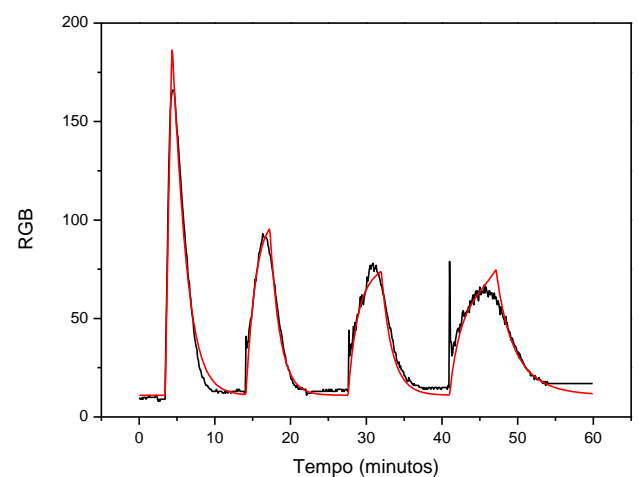

(b)

Figura 4. Comparação de valores de RGB calculados com o modelo proposto (linha vermelha) e os dados experimentais (linha preta) correspondentes ao experimento 2; (b) experimento 3 . 
Analogamente ao experimento anterior, o aumento da temperatura influencia a viscosidade da solução e, consequentemente, o coeficiente de transferência de massa, aumentando a taxa de dissolução do $\mathrm{NaCl}$.

\section{CONCLUSÃO}

Foram obtidos dados experimentais de dissolução de sucessivas adições de $\mathrm{NaCl}$ em soluções contendo diferentes concentrações de MEG. Comparando esses dados experimentais com o modelo cinético desenvolvido, bons resultados foram observados. A partir dos dados experimentais e da modelagem proposta, foram evidenciados e explicitados as influências da temperatura e da concentração de MEG na taxa de dissolução de $\mathrm{NaCl}$.

\section{REFERÊNCIAS BIBLIOGRÁFICAS}

(1) CHAPOY, A.; MAZLOUM, S.; BURGASS, R.; HAGHIGHI, H.; TOHIDI, B. Clathrate hydrate equilibria in mixed monoethylene glycol and electrolyte aqueous solutions, Journal of Chemical Thermodynamics, 48, p.7-12, 2012.

(2) MACCKAY, E., Predicting in situ sulphatescale deposition and the impact on produced íon concentrations, Institution of Chemical Engineers 81, p. 326-332, 2003.

(3) SANDENGEN, K., Prediction of mineral scale formation in wet gas condensate pipelines and in MEG regeneration plants, Doctor Thesis, Tromdheim-NO, 2006.

(4) PRAUSNitZ, J. M., LICHTENTHALER, R. N., AZEVEDO, E. G. Molecular Thermodynamics of Fluid-Phase Equilibra, 3 ed., New Jersey, Prentice Hall PTR, 1999.

(5) SILVA, A., SENA, R., OLIVEIRA, P., BANDINI, T., JUNIOR, A., SENA, R., SILVA, J. Low cost system based on image analysis to determine solubility curve, Sensors and Actuators B, v. 177, p. 1071-1074, 2013.

(6) SILVESTER, L., PITZER, K. Thermodynamics of Electrolytes. 8. High-Temperature Properties, Including Enthalpy and Heat Capacity, with Application to Sodium Chloride. The Journal of Physical Chemistry, v. 81, n. 19, 1977. 